\title{
Usability Evaluation of an Image-based Pill Identification Application
}

\author{
Soo-Kyung Cho ${ }^{1}$, Bora Kim², Eunohk Park ${ }^{2}$, Jieun Kim², Hokyoung Ryu ${ }^{2}$, Yoon-Kyoung Sung ${ }^{1}$ \\ ${ }^{1}$ Department of Rheumatology, Hanyang University Hospital for Rheumatic Diseases, ${ }^{2}$ Department of Arts \& Technology, Hanyang University, \\ Seoul, Korea
}

\begin{abstract}
Objective. To measure the accuracy and usability of an the image-processing based pill identifier application that we have developed. Methods. The subjects selected were medical residents and nurses. Five nurses and five physicians were randomly assigned to use either an the image-processing based pill identifier application $(n=10)$, or the conventional pill identifier application $(n=10)$. They were instructed to examine 10 pills using the application assigned to them, and searches that took $<3$ minutes to find candidate drugs were recognized as successes. Among these successful searches, the accuracy was defined to identify the correct names of the drugs and the times needed in the correctly identifications were also measured. After using one application the subjects were instructed to use the other one and repeat the same process. Finally, they answered a questionnaire on the usability of the applications. Results. The average proportion searches completed within 3 minutes was $91 \%$ for the the image-processing based pill identifier application, slightly, but not significantly, higher than that for the conventional pill identifier application $(85 \%)$. The accuracies of the the image-processing based and conventional pill identifier applications were similar, $89 \%$ and $83 \%$, respectively. In the usability examination, the the image-processing based pill identifier application yielded higher scores for the desirable, usable, findable and useful qualities than the conventional pill identifier application. Conclusion. The the image-processing based pill identifier application application has a similar accuracy to the existing conventional pill identifier application, and its usability was also found to be good. (J Rheum Dis 2019;26:111-117)
\end{abstract}

Key Words. Mobile applications, Pill identification, Usability

\section{INTRODUCTION}

Rheumatic diseases are chronic autoimmune diseases that mostly progress slowly, and are treated with various types of drugs over a long period of time. Treatment often involves so-called combination therapy, in which multiple drugs are taken simultaneously [1]; moreover, many elderly patients take additional drugs for concomitant diseases, which may lead to issues such as polypharmacy $[2,3]$. According to recent reports, polypharmacy is not limited to patients with rheumatic diseases, but has also become a problem in the general population. Almost $60 \%$ of adults in the USA took prescription pills in 2012, up from $50 \%$ in 2000 , and $15 \%$ of adults took 5 or more pre- scription pills in 2012, up from $10 \%$ in 2000 [4]. Along with the increase in the use of drugs, the importance of pill identification associated with patient safety management is also increasing. For example, the number of telephone calls to U.S. Poison Control Centers increased by about $44 \%$ from 2003 to 2007, and the number of calls to identify pills is also on the rise [5].

The importance of pill identification is even more pronounced for inpatients. For instance, when patients with rheumatoid arthritis are hospitalized, no problems will arise if they bring with them the prescription slips from their previous physicians. Since many kinds of drugs are produced by several different pharmaceutical companies, even if they contain the same ingredients it is often neces-

Received : September 18, 2018, Revised : December 6, 2018, Accepted : December 6, 2018

Corresponding to : Yoon-Kyoung Sung iD http://orcid.org/0000-0001-6691-8939

Department of Rheumatology, Hanyang University Hospital for Rheumatic Diseases, 222 Wangsimni-ro, Seongdong-gu, Seoul 04763, Korea. E-mail : sungyk@hanyang.ac.kr 
sary to identify the specific pills that patients have been taking in order to make decisions about treatment. If it is not possible to identify the specific pills they have been taking, serious adverse drug reactions can be caused by excessive drug doses, and diseases can be aggravated by choosing to suspend administration, which may threaten the safety of patients. Drugs that are commonly prescribed can be easily identified by professionals, but physicians with less experience may find it difficult to identify them. For these reasons, there are certain rules requiring pills to have different shapes and colors to avoid confusion between them, and to promote their safe use [6]; also, generic pills are required to differ in shape, color, and size in a manner that differentiates them from the original versions [7].

To prevent a variety of errors and problems caused by unidentifiable pills, identification imprints and registration of drugs are mandatory in Korea, an area that is handled by the Ministry of Food and Drug Safety, and the Korea Pharmaceutical Information Center (KPIC, http:// www.pharm.or.kr/). The program used in Korea to search and identify drugs is based on KPIC data, and users can access the web-based program and extract candidate drugs by directly entering the shape, color and form of pills and the imprints on them. However, it takes a long time to search drugs using this method, and success is highly dependent on the experience of the users who enter the information.

As information technology (IT) has advanced, demand that patients be empowered to self-manage their treatment using IT in order to achieve better outcomes has gradually increased [8]. In the area of rheumatic diseases, terms such as e-rheumatology and e-health are emerging, and attempts are being made to use IT for various purposes [9]. Worldwide, there are approximately 5 billion mobile phone subscribers, and smartphones are constant companions for many individuals [10]. The App Store and Google Play offer around 100,000 health apps, but few have been scientifically evaluated [11]. Mobile health apps could facilitate self-management and adherence to treatment; in addition, they could increase access to care for individuals with limited access, and for those unwilling to seek ordinary health care [12]. Against this background, this study aimed to develop a technology that would identify drugs based on photos of pills taken using a smartphone application. In this way, the current process in which individuals must enter imprinted letters and select the color and form of pills could be avoided, which would reduce the time required for searching, and improve efficiency. It could give consumers a simple way of recognizing mystery pills, help prevent unnecessary medication errors, and reduce waste by identifying pills that might otherwise be discarded.

The purposes of this study were to measure the accuracy of the image-processing based pill identifier application developed here, focusing on drugs for rheumatic diseases, and to examine the usability and utility of the application by comparing its results with the results achieved through searches using the existing conventional pill identifier application.

\section{MATERIALS AND METHODS}

\section{Study design and subjects}

\section{1) Study design}

Randomized controlled trials were used to evaluate the utility of the image-processing based pill identifier application compared to the existing conventional pill identifier application, and the same pills were identified using the two applications.

\section{2) Study subjects}

The study subjects were selected from among medical personnel with relatively little experience in identifying pills: 10 medical residents and 10 nurses (Figure 1). They were randomly assigned to the conventional pill identifier application and to the image-processing based pill identifier application. The random assignment was conducted using the Excel program. The participants voluntarily signed a consent form for participation.

\section{Methods}

1) Applications used to identify pills

Two applications were used. The conventional pill iden-

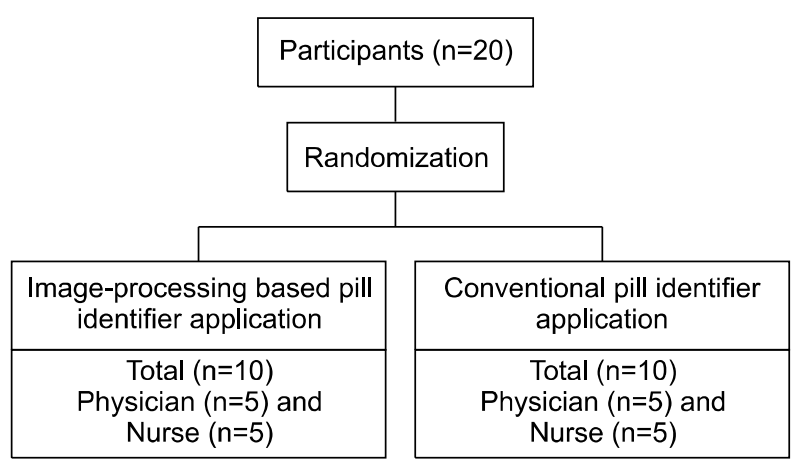

Figure 1. Flow diagram of subjects' enrollment. 
tifier application consisted of the free application provided by KPIC. It offers several services on the main page. When users click the button to "search drugs by shape," they can enter the imprint on a pill (English letter, Korean alphabet, number), and select the form, shape and color of the pill; candidate drugs are then listed on the screen. For the present study, an image-processing based pill identifier application was developed named "ALBOX". When a camera button on the main page is clicked, users can select the form and shape and type of a pill, and take a photo of it, and the application automatically searches its database for the pill. To perform this process, the image-processing based pill identifier application accesses the same database provided by KPIC (Supplementary Figure 1). The imprints on the pill are extracted from the image, and the search results are listed on the screen. When the "OK" button is clicked, candidate drugs are listed. A detailed explanation of this process is presented in Figure 2.

The conventional pill identifier application opens a wide range of search options to ensure better results, which subsequently leads to a long entry process in which the user must type the information in manually. In comparison, the image-processing based pill identifier application, ALBOX, only provides a reduced search option; however, it replaces the tricky manual search process (i.e., typing pill letter and determining colors) with automation. We summarized the comparison between two applications in Supplementary Table 1.
2) Drugs used for measuring the accuracy levels of the applications and time spent searching

In consultation with two rheumatologists, 10 drugs commonly used for rheumatic diseases were selected. Tablet and capsule forms were included (as well as soft and hard capsules) as follows: 4 disease-modifying antirheumatic drugs (DMARDs) and immunosuppressive drugs (Haloxin [hydroxychloroquine sulfate] $200 \mathrm{mg}$, Tacrobel [tacrolimus hydrate] $1 \mathrm{mg}$, Cellcept [mycophenolate mofetil] $250 \mathrm{mg}$, Cipol-N [microemulsion cyclosporine] $25 \mathrm{mg}$ ); 4 anti-inflammatory analgesic drugs (Airtal [aceclofenac], Vimovo [naprexen and esomeprazole], Tridol [tramadol hydrochloride] $50 \mathrm{mg}$, Celebrex [celecoxib] $200 \mathrm{mg}$ ); and 2 anti-osteoporotic agents (Evista [raloxifene HCI] $60 \mathrm{mg}$, Calcio [calcitriol]), as shown in Supplementary Table 2 and Supplementary Figure 2.

\section{3) Measuring the accuracy levels of the applications and time spent searching}

Study subjects were instructed to install on their devices both the conventional and image-processing based pill identifier application, and to search 10 pills that were selected in advance using the assigned application. The time required to identify each pill was measured. Searches that took over 3 minutes were considered to be failures to identify drugs, and the subjects were instructed to search another pill. Those that took less than 3 minutes to be presented with candidate drug identities were recognized as successes in recognizing drugs, and

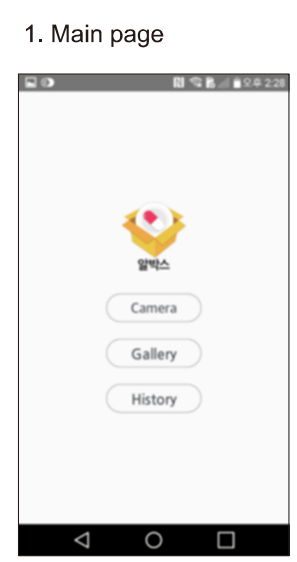

2. Camera

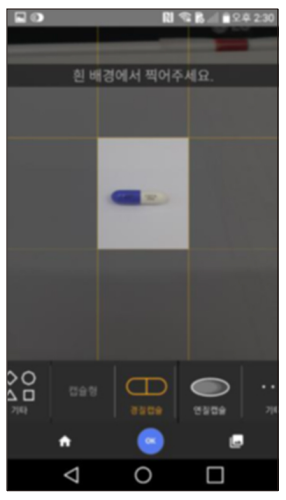

3-1. Imprint extraction results (searching)

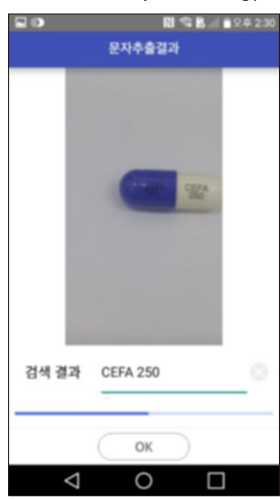

3-2. Imprint extraction results (finished)

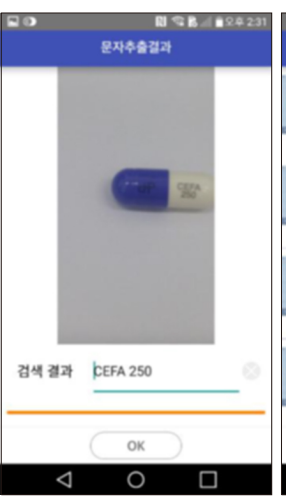

4. Search results

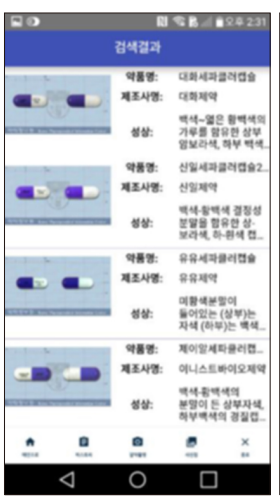

5. Detailed information

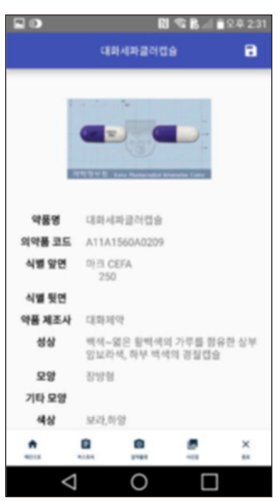

Figure 2. Pill identification by the image-processing based pill identifier application. 1. The main page displays three menu items (camera, gallery, history). 2. When the camera screen is on, users choose the tablet or capsule form and shape of the pill they want to search. 3. The progress of extraction of imprints is displayed in the color bar, and when the bar turns orange (meaning finished), users click the OK button to see the search results. 4. Candidate drugs are listed on the search result screen. 5. When a drug is selected, detailed information on it is presented on the screen. 
the proportion of such instances in which the correct drug was identified was used to evaluate the accuracy of the application. The time spent in the correct identifications were also measured.

\section{4) Evaluating the usability of the pill identifier applications}

Usability refers to the user convenience and efficiency of tangible and intangible goods such as products and services. An evaluation of usability is a process that measures and evaluates interrelationships between users and products or services from the perspective of the users, taking into consideration user environments, and the purpose of the process is to provide the optimal environment for users [13]. Morville has proposed a honeycomb model as a tool to identify whether a solution addresses all facets of the user experience in a holistic manner. The honeycomb model evaluates 7 facets of the user experience, providing a simple and clear structure for ease of understanding [14].

In this study, the central facet of the honeycomb modelvaluable-was excluded, and the other 6 facets that were suitable for this study were reorganized as follows: useful, usable, desirable, findable, accessible, and credible. The questionnaires used were composed of 20 questions, and a 5-point scale was used, as shown in Supplementary Table 3. The higher the score, the more positive was the response. Depending on the average score, responses were classified as follows: very positive $(\geq 4)$; positive $(\geq 3)$; normal $(=2.5)$; negative $(\leq 2)$; and very negative $(\leq 1)$. After conducting the randomly controlled trial, those who used the conventional pill identifier application were instructed to use the image-processing based pill identifier application following the same process, and vice versa. Those who completed these two processes answered the questionnaires on the usability of the two applications.

\section{Statistical analysis}

The demographic characteristics of users in the two groups were compared using Mann-Whitney and chisquare tests. The time spent to identify individual drugs was compared using the Mann-Whitney test, and the correct answer rates of the two groups were compared using the chi-square test. All statistical analyses were conducted using PASW Statistics version 18 (IBM, Armonk, NY, USA). P-values $<0.05$ were regarded as statistically significant.

\section{Ethical approval}

This study was conducted with approval from the Institutional Review Board (IRB) of Hanyang University Hospital (Research No.: HYUH 2017-05-006-002).

Table 1. Recognition and accuracy rates, and times needed to identify accurate drug names

\begin{tabular}{|c|c|c|c|c|c|c|c|c|c|}
\hline \multirow{2}{*}{$\begin{array}{c}\text { Pill } \\
\text { identification } \\
\text { application }\end{array}$} & \multicolumn{3}{|c|}{ Recognition rate* } & \multicolumn{3}{|c|}{ Accuracy rate $^{\dagger}$} & \multicolumn{3}{|c|}{ Time spent to find accurate drug names $^{\ddagger}$} \\
\hline & $\begin{array}{l}\text { Image- } \\
\text { processing } \\
\text { based }\end{array}$ & Conventional & p-value & $\begin{array}{l}\text { Image- } \\
\text { processing } \\
\text { based }\end{array}$ & Conventional & p-value & $\begin{array}{l}\text { Image- } \\
\text { processing } \\
\text { based (s) }\end{array}$ & $\begin{array}{c}\text { Conventional } \\
\text { (s) }\end{array}$ & $\mathrm{p}$-value \\
\hline Drug 1 & 100 & 80 & 0.45 & 100 & 100 & 0.47 & $74.2 \pm 49.9$ & $70.5 \pm 49.3$ & 0.83 \\
\hline Drug 2 & 80 & 80 & 1.00 & 100 & 100 & 1.00 & $53.9 \pm 45.0$ & $41.1 \pm 21.9$ & 0.80 \\
\hline Drug 3 & 90 & 80 & 1.00 & 100 & 100 & 1.00 & $48.9 \pm 32.2$ & $61.6 \pm 41.9$ & 0.89 \\
\hline Drug 4 & 70 & 90 & 0.58 & 85.7 & 100 & 0.27 & $66.3 \pm 43.2$ & $53.7 \pm 47.2$ & 0.36 \\
\hline Drug 5 & 100 & 90 & 1.00 & 100 & 100 & 1.00 & $29.8 \pm 12.1$ & $21.8 \pm 12.8$ & 0.08 \\
\hline Drug 6 & 100 & 100 & - & 90 & 80 & 1.00 & $41.5 \pm 16.3$ & $27.6 \pm 11.1$ & 0.02 \\
\hline Drug 7 & 90 & 70 & 0.58 & 100 & 100 & 0.58 & $36.7 \pm 18.9$ & $64.6 \pm 45.5$ & 0.25 \\
\hline Drug 8 & 90 & 70 & 0.58 & 100 & 100 & 0.58 & $35.7 \pm 12.6$ & $40.6 \pm 34.3$ & 0.25 \\
\hline Drug 9 & 100 & 100 & - & 100 & 100 & - & $25.5 \pm 12.8$ & $18.6 \pm 7.9$ & 0.06 \\
\hline Drug 10 & 90 & 90 & 1.00 & 100 & 100 & 1.00 & $46.0 \pm 26.9$ & $39.2 \pm 21.8$ & 0.67 \\
\hline
\end{tabular}

Values are presented as \% or mean \pm standard deviation. $*$ Recognition rate means the percentage of the searches of the 10 drugs that were completed by the given application within 3 minutes. ${ }^{\dagger}$ Accuracy rate means the percentage of the searches of the 10 drugs completed within 3 minutes that correctly identified the drug. ${ }^{\ddagger}$ The mean time needed to identify each drug whose name was accurately identified by the given application. 


\section{RESULTS}

\section{Demographic and clinical characteristics of subjects}

The average age of the 20 subjects was 28.5 years (standard deviation $=4.0$ ), and 13 were females $(65 \%)$. There were no statistically significant differences in age (28.6 \pm 4.2 vs. $28.3 \pm 2.8)$ and gender ( $80 \%$ vs. $50 \%$ ) between the conventional pill identifier application group and the image-processing based pill identifier application group $(p=0.88)$.

\section{Measuring the accuracy of the image-processing based pill identifier application and the time spent searching}

Both applications were satisfactory (over 70\%) in terms recognizing the 10 drugs. The image-processing based pill identifier application had a higher recognition rate than the conventional pill identifier application for all drugs with the exception of one (Cipol-N [microemulsion cyclosporine] $250 \mathrm{mg}$ ), but the difference was not statistically significant. In terms of the accuracy of identification, the two applications gave similar results when it came to the proportion of correct drug identifications among those searches completed within 3 minutes. The accuracy of the image-processing based pill identifier application for (Cipol-N [microemulsion cyclosporine] $250 \mathrm{mg}$ ) was relatively low, but the difference between the two applications was not statistically significant. In terms of searching times, the image-processing based pill identifier application took slightly longer to search Drug 6 (Vimovo [naprexen and esomeprazole]) (41.5 \pm 16.3 vs. $27.6 \pm$ 11.1, $\mathrm{p}=0.02$ ), but in other drugs, the difference between the two applications was not statistically significant (Table 1).

\section{Usability of the image-processing based pill iden- tifier application}

The results of the evaluation of the usability of the two applications are shown in Figure 3. Overall, the image-processing based pill identifier application gave higher scores for the desirable, usable, findable and useful qualities than the conventional pill identifier application, and in particular, had a very high score (4.2 points) for desirable. Both applications had scores of normal for credible, the score of the conventional pill identifier application being 2.7 points, slightly higher than that of the image-processing based pill identifier application. Both applications gave positive results (3.0 points) for acces- sible, and there was no statistically significant difference between them (Figure 3).

\section{DISCUSSION}

The image-processing based pill identifier application developed in this study yielded similar results to the existing conventional pill identifier application in terms of the recognition rate of drugs used for rheumatic diseases, and time spent searching drugs. The results of the usability evaluation of the image-processing based pill identifier application were higher in the desirable, usable and findable categories than the existing application, but the score for credible was not satisfactory. Each question on usability was reviewed in detail, and it was found that errors occurred frequently in the use of the application, and that the reasons for such errors were not immediately explained to users, which seems to be the reason why the unsatisfactory score was given for credible.

The recognition and accuracy rates of the image-processing based pill identifier application were very high when letters were clearly printed and recorded on pills. The accuracy of the application developed in this study was relatively low for Cipol-N (microemulsion cyclosporine) $250 \mathrm{mg}$, which is attributable to the form of the pill. It was difficult to recognize the imprints on the soft capsule of Cipol-N (microemulsion cyclosporine) 250 $\mathrm{mg}$, and this seemed to be responsible for the low recog-
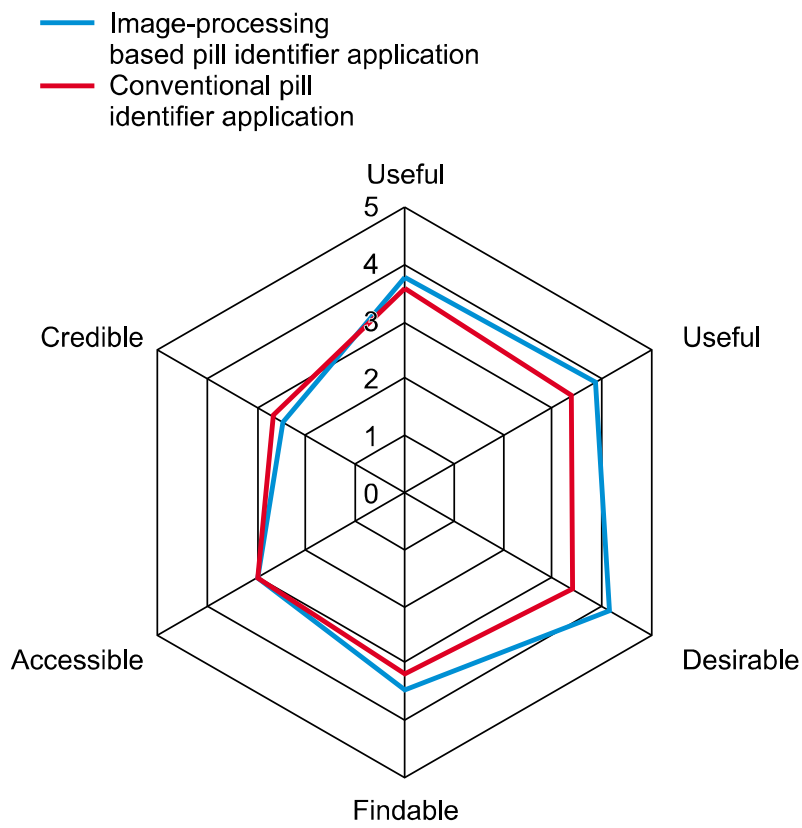

Figure 3. Usability test results for the two applications. 
nition and accuracy rates. In a test conducted on various drugs prior to this study, it was found that the recognition rate of letters engraved on pills was highly dependent on the surrounding environment (light and shadow, background, etc.), and was thus relatively low. An additional study is currently being conducted to address this issue, in order to develop a device that can shed light from various angles, and thus increase the recognition rate of the application.

This study is the first attempt both in Korea and abroad to develop an application using an image-processing based pill identification technology, and to evaluate the usability of the application. Several previously developed applications for identification of pills search for a pill based on user input information. On the other hand, the newly developed image-processing based pill identification searches for a pill based on information objectified by taking a photo. A method for objectively recognizing the shape and color of a drug, which individual users can judge differently, can reduce the error by users. In addition, this image-processing based pill identification is more intuitive, in that it is input by taking a photo, while conventional pill identifier applications are based on drug information entered one by one in order. Attempts have been gradually made in the field of rheumatology to create improved treatment environments, and to obtain better disease outcomes by applying IT and electronic devices $[8,15]$. The image-processing based pill identifier application developed in this study is expected to improve medical service environments, and to provide a positive example of the empowerment of patients.

Meanwhile, the image-processing based pill identifier application has some limitations, such as low recognition rates for some forms of pills, but by using it, medical personnel can search drugs that patients have taken in a quick and convenient manner by exploiting the accuracy and convenience of the application. In addition, it should be noted that the results we obtained from the well-controlled environment for clinical studies may not fully reflect the results of use in a general environment. Another limitation of this study was that only drugs commonly employed to treat rheumatic diseases were used to evaluate the recognition and accuracy rates of the application. We tried to select a range of forms, colors and shapes, but it will be important to test a broader range of drugs. However, a more careful approach and evaluation should be conducted, particularly when using look-alike drugs. The results of this study suggest that the new applica- tion will address some of the difficulties and limitations of the medical environment. For example, in the existing procedures, the drugs that inpatients bring with them are delivered to the pharmacy department and have to be personally identified by professional pharmacists using website searches. The new application can be employed by nurses and physicians in wards, as it is not highly dependent on the work experience of individual users. With this application, the time required to recognize drugs can be shortened, drugs can be identified regardless of location and device using smartphones, and information on the searched drugs can be easily obtained. Nevertheless, it will be important to improve the accuracy of identification, and to connect the application with the database provided by the KPIC for continuous use.

From a long-term perspective, it will be desirable to improve and strengthen the credibility (suggesting reasons for errors and countermeasures) and accessibility (improving the help service), which were found to be weak in the usability evaluation of the application. It will also be good to extend the service to the general public, not just medical personnel. To do so, the accuracy of drug identification needs to be improved, and safeguards need to be implemented to prevent inaccurate identification results. In particular, artificial intelligence (AI) can be used to improve accuracy. In addition, for patients, it will be helpful to add the ability to keep records of the medication prescribed to them, and to see whether this improves drug adherence. The application can be used not only to identify drugs for rheumatic diseases; it can also be extended to manage chronic diseases for which a number of drugs have to be administered continuously, and infectious diseases whose outcomes are highly dependent on drug adherence.

\section{CONCLUSION}

In conclusion, an image-processing based pill identifier application has been developed that can be used on smartphones. The developed application had a similar level of accuracy to the existing conventional pill identifier application when used by medical personnel, and its usability was also found to be good. In the future, it will be important to extend the usability of the application to the general population, and to update the database to increase its accuracy. 


\section{ACKNOWLEDGMENTS}

This research was supported by a grant from the Korea Health Technology R\&D Project through the Korea Health Industry Development Institute (KHIDI), funded by the Ministry of Health \& Welfare, Republic of Korea (grant number: HI15C1102).

\section{CONFLICT OF INTEREST}

No potential conflict of interest relevant to this article was reported.

\section{SUPPLEMENTARY DATA}

Supplementary data can be found with this article online at https://doi.org/10.4078/jrd.2019.26.2.111.

\section{REFERENCES}

1. Smolen JS, Landewé R, Bijlsma J, Burmester G, Chatzidionysiou K, Dougados $\mathrm{M}$, et al. EULAR recommendations for the management of rheumatoid arthritis with synthetic and biological disease-modifying antirheumatic drugs: 2016 update. Ann Rheum Dis 2017; 76:960-77.

2. Treharne GJ, Douglas KM, Iwaszko J, Panoulas VF, Hale ED, Mitton DL, et al. Polypharmacy among people with rheumatoid arthritis: the role of age, disease duration and comorbidity. Musculoskeletal Care 2007;5:175-90.

3. Wolf MS, Curtis LM, Waite K, Bailey SC, Hedlund LA, Davis TC, et al. Helping patients simplify and safely use complex prescription regimens. Arch Intern Med 2011;171:300-5.

4. Kantor ED, Rehm CD, Haas JS, Chan AT, Giovannucci EL. Trends in prescription drug use among adults in the United States from 1999-2012. JAMA 2015;314:1818-31.
5. Spiller HA, Griffith JR. Increasing burden of pill identification requests to US Poison Centers. Clin Toxicol (Phila) 2009;47:253-5.

6. Hellier E, Tucker M, Kenny N, Rowntree A, Edworthy J. Merits of using color and shape differentiation to improve the speed and accuracy of drug strength identification on over-the-counter medicines by laypeople. J Patient Saf 2010;6:158-64.

7. Greene JA, Kesselheim AS. Why do the same drugs look different? Pills, trade dress, and public health. N Engl J Med 2011;365:83-9.

8. Steinhubl SR, Muse ED, Topol EJ. Can mobile health technologies transform health care? JAMA 2013;310:2395-6.

9. El Miedany Y. e-Rheumatology: are we ready? Clin Rheumatol 2015;34:831-7.

10. World Health Organization. From innovation to implementation: eHealth in the WHO European region [Internet]. Copenhagen: World Health Organization, 2016 [cited 2017 Aug 22]. Available from: http://www.euro.who.int/_data/assets/pdf_file/0012/302331/From-Innovationto-Implementation-eHealth-Report-EU.pdf?ua =1.

11. Whitehead L, Seaton P. The effectiveness of self-management mobile phone and tablet apps in long-term condition management: a systematic review. J Med Internet Res 2016; 18:e97.

12. Hamine S, Gerth-Guyette E, Faulx D, Green BB, Ginsburg AS. Impact of mHealth chronic disease management on treatment adherence and patient outcomes: a systematic review. J Med Internet Res 2015;17:e52.

13. Woo KH. Comparison of the usability of microblog - focused on the comparison between the Twitter and me2DAY -. J Digit Design 2010;10:463-73.

14. Kwon GM, Kim AR, Kim SI. The activation methods of mobile service through usability testing - focus on comparison mobile application YES24 and KYOBO Bookstores. J Digit Design 2014;14:392-9.

15. Collins TR. Tech talk: apps put more rheumatology information at fingertips [Internet]. Atlanta: American College of Rheumatology, 2012 Jun 10 [cited 2017 Aug 22]. Available from: https://www.the-rheumatologist. org/article/tech-talk-apps-put-more-rheumatology-informationat-fingertips/. 
pISSN: 2093-940X, elSSN: 2233-4718

Journal of Rheumatic Diseases Vol. 26, No. 2, April, 2019

https://doi.org/10.4078/jrd.2019.26.2.111

Supplementary Table 1. The comparison between the conventional pill identifier and image-processing based pill identifier application

\begin{tabular}{lcc}
\hline \hline \multicolumn{1}{c}{ Characteristics of pill } & $\begin{array}{c}\text { Conventional pill } \\
\text { identifier application }\end{array}$ & $\begin{array}{c}\text { Image-processing based pill } \\
\text { identifier application }\end{array}$ \\
\hline Color of pill & Manual & Automated \\
Letter on the surface of pill & Manual & Automated \\
Shape of pill & Manual & Manual \\
Type of pill & Manual & Manual \\
Special character on the surface of pill & Manual & N/A \\
Manufacturer & Manual & N/A \\
\hline
\end{tabular}

N/A: not available. 
Soo-Kyung Cho et al.

Supplementary Table 2. Ten drugs of different types selected for the test

\begin{tabular}{lll}
\hline \hline \multicolumn{1}{c}{ Drug class } & Brand name of drug & \multicolumn{1}{c}{ Generic name of drug } \\
\hline Disease modifying anti-rheumatic/ & Haloxin $200 \mathrm{mg}$ & Hydroxychloroquine sulfate \\
immunosuppressive drugs & Tacrobel $1 \mathrm{mg}$ & Tacrolimus hydrate \\
& Cellcept $250 \mathrm{mg}$ & Mycophenolate mofetil \\
& Cipol-N $25 \mathrm{mg}$ & Microemulsion cyclosporine \\
Anti-inflammatory analgesic drugs & Airtal & Aceclofenac \\
& Vimovo & Naprexen and esomeprazole \\
& Tridol $50 \mathrm{mg}$ & Tramadol hydrochloride \\
Anti-osteoporotic agents & Celebrex $200 \mathrm{mg}$ & Celecoxib \\
& Evista $60 \mathrm{mg}$ & Raloxifene $\mathrm{HCl}$ \\
\hline
\end{tabular}


Supplementary Table 3. Questionnaire for evaluating usability based on the classification system of the honeycomb model

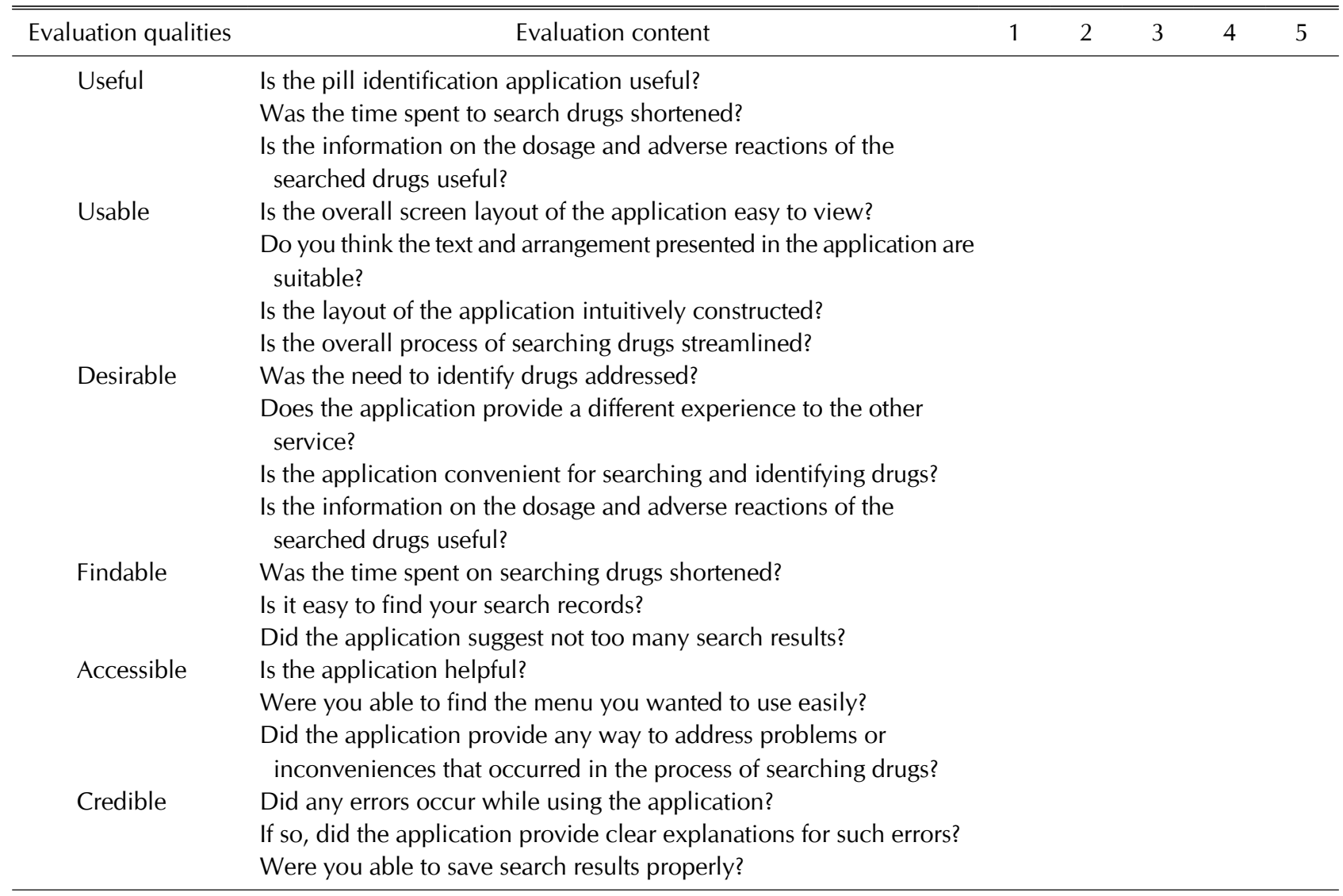




\section{Smartphone}

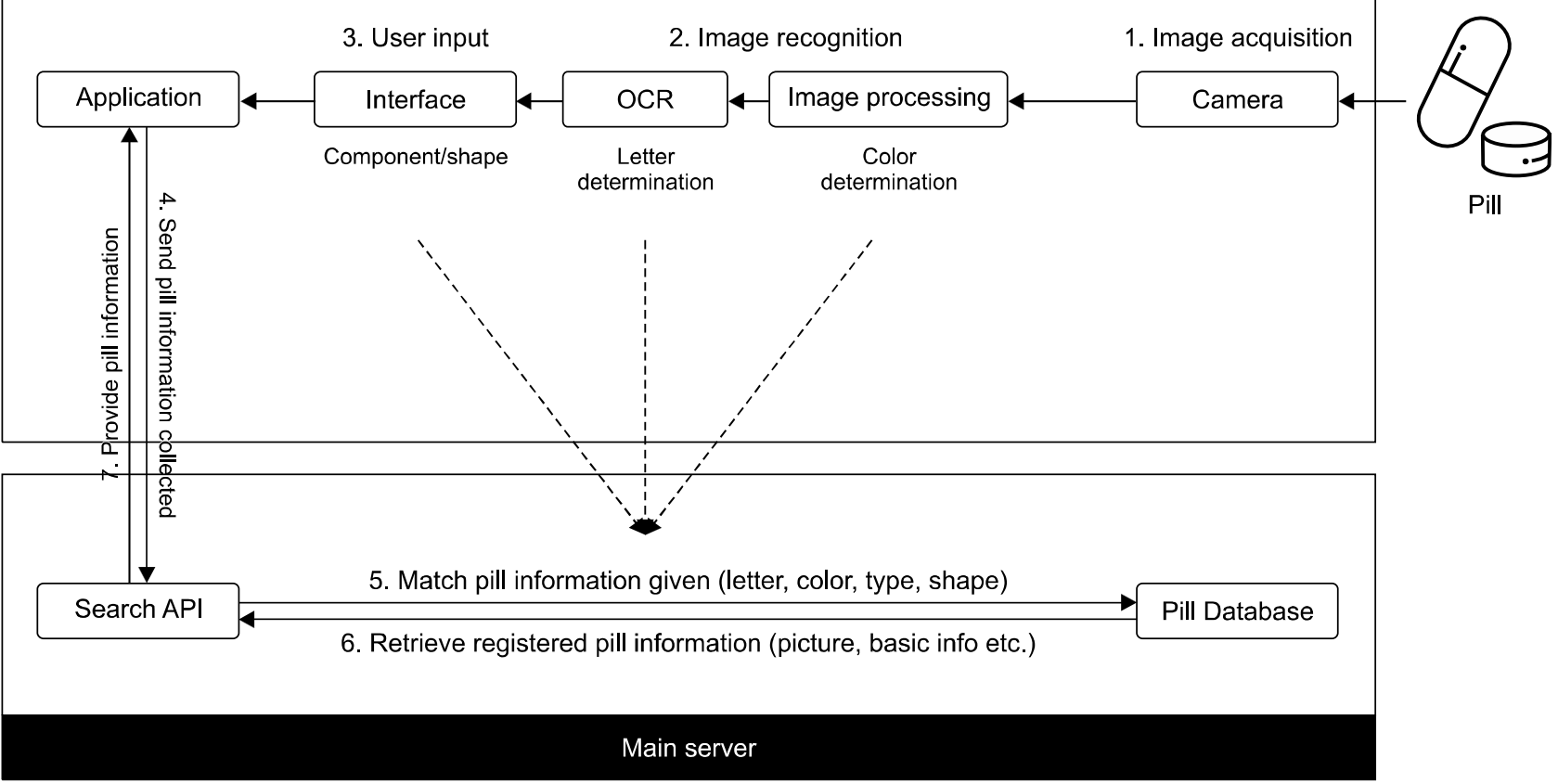

Supplementary Figure 1. System working module of image-processing based pill identifier application. OCR: optical character recognition, API: application programming interface. 


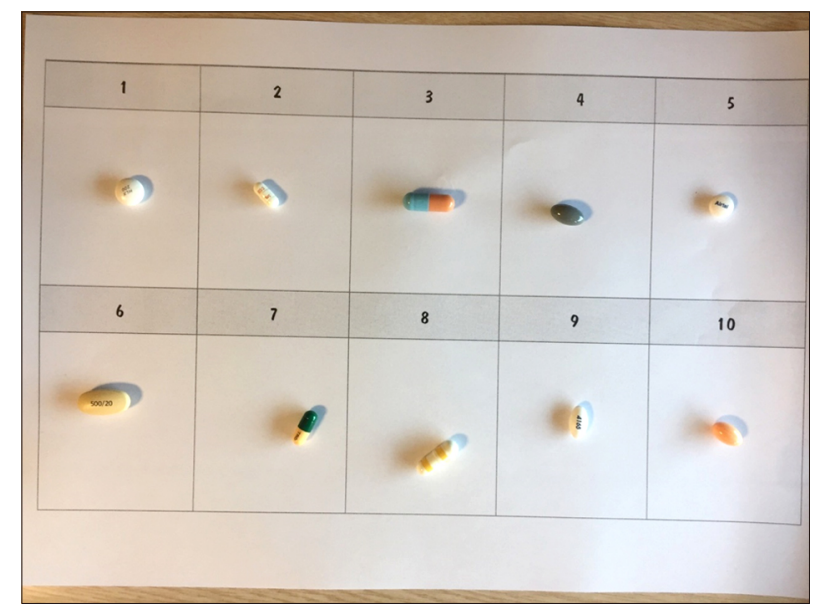

Supplementary Figure 2. The ten drugs of different types selected for the test (ready for the test). 